\title{
Isolation And Structural Characteristic Of Novel Gangliosides From Temnopeuridae Echnoid Gonads With Investigation On Their Mouth And Breast Anticancer Properties $^{\dagger}$
}

\author{
R. Rajeswari ${ }^{1, *}$, A. Priyanka ${ }^{1}$, V. Vaishnavidevi ${ }^{1}$ \\ 1 Department of Biotechnology, P.S.R. Engineering College, Sivakasi, Tamil Nadu, India \\ * Correspondence: raajiramesh80@ gmail.com; \\ $\uparrow$ Presented at International e-Conference on Bioengineering for Health and Environment (ICBHE 2020)
}

Received: 5.07.2020; Revised: 10.07.2020; Accepted: 12.07.2020; Published: 15.07.2020

\begin{abstract}
Sea urchins have recently been attracting considerable research interest as sources of a number of highly valuable bioactive compounds that possess antitumor, antiviral, antimicrobial and anticoagulant properties that hold great promise for use in pharmacological applications. The present study was carried out to determine the structural, developmental characterization of gangliosides isolated from the Temnopeuridae. The extraction of lipids carried out by the chloroform-methanol extraction methods. The structural identification of Temnopeuridae extracts determined by TLC, GCMS, FT-IR, and NMR studies. The antimicrobial and antifungal activity of the Temnopeuridae extract against the various clinical pathogens were analyzed. Then the in-vitro studies were carried out in HELA cells. In the experimental results, a significant amount of lipids were extracted from the gonods of Temnopeurida. The presence of polyunsaturated fatty acids and enriched phospholipid profile were identified. The clinical pathogens such as Clostridium, Staphylococcus, Vibrio species, Candida, Aspergillus, and mucormycets showed a potential zone of inhibition during the disc diffusion method. The cytotoxicity assay showed an increase in cell viability against the cytotoxicity in HELA cells. Based on the consideration of the high level of polyunsaturated fatty acids and enriched phospholipids, Temnopeurida gonads offer novel therapeutic compounds for the mouth and breast cancer.
\end{abstract}

Keywords: Temnopeurida; Sea urchin; anticancer activity; antimicrobial activity.

(C) 2020 by the authors. This article is an open-access article distributed under the terms and conditions of the Creative Commons Attribution (CC BY) license (https://creativecommons.org/licenses/by/4.0/).

\section{Funding}

This research received no external funding.

\section{Acknowledgments}

This research has no acknowledgment.

\section{Conflicts of Interest}

The authors declare no conflict of interest. 